\title{
Abdominal muscle activity during breathing with and without inspiratory and expiratory loads in healthy subjects

\author{
António Mesquita Montes ${ }^{\mathrm{a} b \mathrm{~b}}$, João Baptista ${ }^{\mathrm{c}}$, Carlos Crasto ${ }^{\mathrm{a}}$, Cristina Argel de Melo ${ }^{\mathrm{a}}$, Rita Santos ${ }^{\mathrm{a}}$, \\ João Paulo Vilas-Boas ${ }^{b}$
}

\author{
a Department of Physiotherapy, and Activity and Human Movement Study Center (CEMAH), School of Allied Health Technologies, Polytechnic Institute of Porto, Rua Valente \\ Perfeito 322, 4400-330 Vila Nova de Gaia, Portugal \\ ${ }^{\mathrm{b}}$ Faculty of Sport, CIFI2D, and Porto Biomechanics Laboratory (LABIOMEP), University of Porto, Rua Dr. Plácido Costa 91, $4200-450$ Porto, Portugal \\ ${ }^{\mathrm{c}}$ Physiotherapist, Private Practice, Portugal
}

\begin{abstract}
A B S T R A C T
Central Nervous System modulates the motor activities of all trunk muscles to concurrently regulate the intra-abdominal and intra-thoracic pressures. The study aims to evaluate the effect of inspiratory and expiratory loads on abdominal muscle activity during breathing in healthy subjects. Twenty-three higher education students $(21.09 \pm 1.56$ years; 8 males) breathed at a same rhythm (inspiration: two seconds; expiration: four seconds) without load and with $10 \%$ of the maximal inspiratory or expiratory pressures, in standing. Surface electromyography was performed to assess the activation intensity of rectus abdominis, external oblique and transversus abdominis/internal oblique muscles, during inspiration and expiration. During inspiration, transversus abdominis/internal oblique activation intensity was significantly lower with inspiratory load when compared to without load $(p=0.009)$ and expiratory load $(p=0.002)$. During expiration, the activation intensity of all abdominal muscles was significantly higher with expiratory load when compared to without load $(p<0.05)$. The activation intensity of external oblique $(p=0.036)$ and transversus abdominis/internal oblique $(p=0.022)$ was significantly higher with inspiratory load when compared to without load. Transversus abdominis/internal oblique activation intensity was significantly higher with expiratory load when compared to inspiratory load $(p<0.001)$.

Transversus abdominis/internal oblique seems to be the most relevant muscle to modulate the intraabdominal pressure for the breathing mechanics.
\end{abstract}

Keywords: Respiration; Postural control; Core abdominal; Respiratory loads; Surface electromyographic activity

\section{Introduction}

The constant change of internal and external forces acting on human body determines that the Central Nervous System (CNS) organizes a complex and differentiated control and coordination system, allowing movement without losing stability (Horak et al., 1997). CNS continuously interprets the stability status, receiving and integrating afferent input from the peripheral mechanoreceptors and other sensory systems, to generate a coordinated response of trunk muscles (Balasubramaniam and Wing, 2002; Lackner and DiZio, 2005). The global and core muscles provide stability to the multi segmental spine by modulating the intra-abdominal pressure (Cholewicki et al., 1999), which occurs through the coordination of abdominal, pelvic floor and diaphragm muscles activity (Hodges et al., 1997a; Hodges and Gandevia, 2000a).
Nevertheless, CNS modulates the motor activities of these trunk muscles during both postural and respiratory functions (Hodges, 1999) to concurrently regulate the intra-abdominal and intrathoracic pressures (Hodges et al., 2001). The postural control inputs from the supraspinal structures are integrated with the rhythmic drive to the inspiratory motoneurons of diaphragm muscle from the respiratory centres in the pons and medulla. Although rectus abdominis (RA) and external oblique (EO) muscles are a non respiration-related modulation, the transversus abdominis ( $\operatorname{Tr} A)$ muscle activity is modulated with the respiration and is out of phase with the diaphragm muscle activity. Then, the tonic activity of diaphragm and TrA muscles for the postural control is modulated with the respiratory phasic activity (Hodges and Gandevia, 2000a, 2000b). 
During tidal breathing, the diaphragm muscle contraction causes a descent of its dome and, consequently, an increase in intra-abdominal pressure, which eventually prevents a further descent of the central tendon of diaphragm muscle (Goldman et al., 1987). This increased intra-abdominal pressure is countered by the tension in the abdominal muscles, namely TrA muscle (De Troyer et al., 1990). Without sufficient compliance in abdominal muscles, the central tendon of diaphragm muscle cannot be effectively stabilized to promote lower ribs elevation and, consequently, lateral chest wall expansion (De Troyer and Estenne, 1988).

The breathing movements of rib cage and abdomen generate a cyclical disturbance to the trunk stability and the body equilibrium (Hodges et al., 2002). Despite healthy subjects are capable of actively compensate for the quiet breathing, postural control is compromised when the respiratory demand increases and requires voluntary control (David et al., 2012; Kuznetsov and Riley, 2012). This increased descending respiratory drive attenuates the postural activity of diaphragm and TrA muscles (Hodges et al., 2001). The impaired contribution of these core muscles to the spinal stability is associated with an increased activity of EO and RA muscles (Hodges and Gandevia, 2000b). Furthermore, the respiratory system tends to limit the inspiratory muscle activity, transferring any additional load to the expiratory muscles, placing the diaphragm muscle in improved mechanical advantage, to assist the subsequent inspiration (Aliverti et al., 1997). Nevertheless, the impact of different respiratory loads on abdominal muscle activity, during both breathing phases, for the synchronization of postural and respiratory functions is not yet clear. Thus, the aim of the present study was to evaluate the effect of inspiratory and expiratory loads on abdominal muscle activity during breathing, in healthy subjects. Specifically, it was analysed the activation intensity of RA, EO and transversus abdominis/internal oblique (TrA/IO) muscles, during inspiration and expiration, without respiratory load and with inspiratory or expiratory loads.

\section{Methods}

\subsection{Sample}

The study followed a repeated measures design with a sample composed by twenty-three healthy higher education students, who volunteered to participate in this research (8 males). Demographic and anthropometric data regarding the sample are described in Table 1. Participants were aged between 18 and 24 years and were not participated in moderate intensity, aerobic physical activity for a minimum of $30 \mathrm{~min}$ on five days a week or vigorous intensity, aerobic activity for a minimum of 20 min on 3 days a week, for a period exceeding one year (Thompson, 2014). It was defined as exclusion criteria the body mass index higher than $25 \mathrm{~kg} \mathrm{~m}^{-2}$; chronic nonspecific lumbopelvic pain (recurrent episodes of lumbopelvic pain for a period longer than three months); scoliosis, length discrepancy of the lower limbs or other postural asymmetries; history of spinal, gynaecological or abdominal surgery in the previous year; neurological or inflammatory disorders; metabolic or cardio-respiratory diseases; pregnancy or post-delivery in the previous six months; smoking

Table 1

Sample characterization: demographic and anthropometric data, with mean, standard deviation, minimum and maximum.

\begin{tabular}{lrlll}
\hline & Mean & Standard deviation & Minimum & Maximum \\
\hline Age (years) & 21.09 & 1.56 & 18 & 24 \\
Body mass $(\mathrm{kg})$ & 61.57 & 8.87 & 49 & 81 \\
Height $(\mathrm{m})$ & 1.67 & 0.90 & 1.53 & 1.82 \\
BMI $\left(\mathrm{kg} \mathrm{m}^{-2}\right)$ & 21.96 & 1.51 & 19.02 & 24.45 \\
\hline
\end{tabular}

BMI body mass index habits; long-term corticosteroid therapy; and any conditions that may interfere with the data collection (American Thoracic Society/European Respiratory, 2002; Beith et al., 2001; Chanthapetch et al., 2009; Hermens et al., 2000; Mew, 2009; Reeve and Dilley, 2009). Each participant provided a written informed consent form, according to the Declaration of Helsinki. The anonymity of participants and the confidentiality of data were guaranteed. The Institutional Research Ethics Committee previously approved this study.

\subsection{Procedures}

\subsubsection{Sample selection and characterization}

An electronic questionnaire was delivered to all participants to verify the selection criteria and to collect sociodemographic information. Anthropometric measures were assessed in participants who fulfil participation criteria. Height $(\mathrm{m})$ and body mass $(\mathrm{kg})-$ were measured using a seca 222 stadiometer with an accuracy of $1 \mathrm{~mm}$ and a seca 760 scale with an accuracy of $1 \mathrm{~kg}$, respectively, and then used to calculate the body mass index. To assess postural asymmetries, the lower limb length $(\mathrm{cm})$ was measured using a seca 201 tape with an accuracy of $1 \mathrm{~mm}$ (seca - Medical Scales and Measuring Systems, Hamburg, Germany) and the postural assessment was performed. These evaluations were performed to select the final sample. Women who were in luteal phase were contacted later for data collection.

\subsubsection{Data collection protocol}

The study procedures took place at a biomechanical laboratory and were performed in a controlled environment. To avoid interrater error, each researcher was responsible for an only task.

Surface electromyography (sEMG) was performed to bilaterally assess the muscle activity of $\operatorname{Tr} \mathrm{A} / \mathrm{IO}, \mathrm{EO}$ and RA. The muscle activity was collected using the BioPlux research device (Plux wireless biosignals S.A., Arruda dos Vinhos, Portugal), with analogue channels of 12 bits and a sampling frequency of $1000 \mathrm{~Hz}$, using double differential electrode leads. To perform the sEMG, the hair was shaved and an abrasive cream was used to remove the dead cells from the skin's surface. Skin was then cleaned with isopropyl alcohol (70\%), removing its oiliness and holding the dead cells. An electrode impedance checker (Noraxon Corporate, Scottsdale AZ, United States of America) was used to make sure that the impedance levels were below $5 \mathrm{~K} \Omega$ and thus ensure a good acquisition of sEMG signal (Hermens et al., 2000). Disposable, self-adhesive $\mathrm{Ag} / \mathrm{AgCl}$ dual snap electrodes (Noraxon Corporate, Scottsdale AZ, United States of America) were used for the sEMG. The electrode characteristics were $4 \times 2.2 \mathrm{~cm}$ of adhesive area, $1 \mathrm{~cm}$ diameter of each circular conductive area and $2 \mathrm{~cm}$ of inter-electrode distance. These electrodes were connected to bipolar active sensors emgPLUX, with a gain of 1000 , an analogue filter at $25-500 \mathrm{~Hz}$ and a common-mode rejection ratio of $110 \mathrm{~dB}$. As the reference electrode, it was used a disposable self-adhesive $\mathrm{Ag} / \mathrm{AgCl}$ snap electrode (Noraxon Corporate, Scottsdale AZ, United States of America) for the sEMG, with $3.8 \mathrm{~cm}$ diameter of circular adhesive area and $1 \mathrm{~cm}$ diameter of circular conductive area. The self-adhesive electrodes were placed in standing, five minutes after the skin preparation. These electrodes were placed parallel to the muscle fibers orientation, according to the references described in Table 2 (Criswell, 2011; Marshall and Murphy, 2003). The electrode placements were confirmed by palpation and muscle contraction. The reference electrode was placed in the anterior superior iliac spine of the contralateral dominant side. The sensors were Bluetooth connected through the sEMG device to a laptop. It was used the MonitorPlux software, version 2.0, to display and acquire the sEMG signal. All electrodes were tested to control the cross-muscular signal (cross-talk), electrical noise and other interferences of sEMG 
Table 2

Recommendations for the electrode placements of rectus abdominis (RA), externa oblique (EO) and transversus abdominis/internal oblique (TrA/IO) muscles.

\begin{tabular}{ll}
\hline Muscle & Anatomical landmarks \\
\hline RA & $\begin{array}{l}2 \mathrm{~cm} \text { lateral to umbilicus, over the muscle mass } \\
\text { EO }\end{array}$ \\
$\begin{array}{l}\text { Lateral to the RA and directly above the anterior superior iliac, } \\
\text { halfway between the crest and ribs at a slightly oblique angle }\end{array}$ \\
$\begin{array}{l}2 \mathrm{~cm} \text { medially and below to anterior superior iliac spine } \\
\text { In this local, TrA and inferior IO muscle fibers are mixed, so it is } \\
\text { impossible distinguish the surface electromyographic activity of } \\
\text { both }\end{array}$
\end{tabular}

signal (Hermens et al., 2000). For this quality control, the baseline and power spectrum of sEMG signal were analysed. It is guaranteed that the baseline values were below $3.5 \mu \mathrm{V}$. Also, the power spectrum curves decreased and reached zero between $200 \mathrm{~Hz}$ and $250 \mathrm{~Hz}$ and atypical power peaks was not present.

A respiratory pressure meter MicroRPM (CareFusion Corporation, San Diego CA, United States of America) was used to assess the maximal inspiratory (MIP) and expiratory (MEP) pressures. The values obtained were used to determine the inspiratory and expiratory loads, respectively. MEP quasi-static maximal manoeuvre was used to normalize the sEMG signal of abdominal muscles (maximal muscle activity of each muscle during breathing). MIP and MEP were both performed with the participants in standing, using a bacterial filter AFT1 - Disposable Bacterial Filter, $22 \mathrm{~mm}$ and a mouthpiece AFT2 - Disposable Mouthpiece, $22 \mathrm{~mm}$ firmly held around the lips to prevent leakage and to support the cheeks, as well as a nasal clip AFT3 - Disposable Noseclip (Biopac Systems Inc., Goleta CA, United States of America) to prevent nasal breathing. To assess MIP, it was performed a forceful and maximal inspiration - Muller manoeuvre - at residual volume; in turn, MEP was assessed through a forceful and maximal expiration - Valsalva manoeuvre - at total lung capacity. Each manoeuvre was encouraged verbally. These manoeuvres were performed during six seconds, with a resting time of three minutes. To calculate the respiratory load, three reproducible manoeuvres were selected, according to the American Thoracic Society/European Respiratory (2002) standards. It was used $10 \%$ of the best value of MIP and MEP for the inspiratory and expiratory loads, respectively.

All participants were submitted to three different tasks breathing without respiratory load and with inspiratory or expiratory loads, in standing - in a single data collection moment. The data collection moment started with breathing without respiratory load. The order of inspiratory or expiratory loads was randomized. A respiratory flow transducer TSD117 - Medium Flow Trans $300 \mathrm{~L} \mathrm{~min}^{-1}$, connected to an amplifier DA100C - General Purpose Transducer Amplifier Module, was used to detect the breathing phases. The respiratory flow was collected using the Biopac MP100WSW Data Acquisition System device (Biopac Systems Inc., Goleta CA, United States of America), with a sampling frequency of $100 \mathrm{~Hz}$. A bacterial filter AFT1, a mouthpiece AFT2 and a nose clip AFT3 were also used. It was used the Acqknowledge software, version 4.1, into display and acquire the respiratory flow signal. Biopac MP100WSW Data Acquisition System was synchronized with the BioPlux research. Thresholds IMT and PEP (Respironics Inc., Murrysville PA, United Stare of America) were used to apply the inspiratory and expiratory loads, respectively. These devices were adapted to the respiratory flow transducer.

The participants were barefoot and had the upper limbs along the body, with feet shoulder-width apart and knees in loose pack position. An A3 paper sheet was used to outline the base of support, keeping it for all tasks. The participants kept their gaze in a horizontal direction and the respiratory flow transducer was kept perpendicular to the participant during all tasks. A single repetition of each task was performed for ten consecutive respiratory cycles, with a resting time of three minutes. The respiratory rhythm (inspiratory time: two seconds; expiratory time: four seconds) was marked through a recorded voice. The participants experienced this respiratory rhythm prior to collect data.

After the data collection, the electrodes were removed and a moisturizing cream was applied on the electrode placements.

\subsubsection{Data processing}

A routine was developed in MatLab Student software (MathWorks, Pozuelo de Alarcon, Spain) to synchronize and process data. Firstly, the sEMG signal was converted into volts. It was applied to the sEMG signal a 2nd order digital filter Infinite Impulse Response - Butterworth, one of $30 \mathrm{~Hz}$ (high pass) and another of $500 \mathrm{~Hz}$ (low pass), to remove the electrical noise and/or cable movement and to remove the cardiac signal. Root mean square (RMS) to 10 samples was then calculated.

Acqknowledge software, version 4.1, was used to analyse data. The abdominal muscle activity was analysed during inspiration and expiration, independently. These both breathing phases were identified through the respiratory flow transducer signal. For the ten respiratory cycles collected, the mean RMS of four central respiratory cycles of each muscle was analysed in each task, with a posterior analysis of its average (Fig. 1).

The muscle activity collected during the MEP manoeuvre was used to normalize data of the abdominal muscles. It was analysed the mean RMS of three central seconds of the expiratory phase of each muscle, and then the average of the mean RMS of three reproducible manoeuvres was calculated (Fig. 2). The percentage of the activation intensity of each muscle was determined according to the following equation:

Muscle activation intensity $(\%)=\left(\frac{\text { mean RMS of each task }}{R M S \text { of the MEP }}\right) * 100$

For the global analysis of activation intensity of the RA, EO and $\mathrm{TrA} / \mathrm{IO}$ muscles during each breathing phase, the average of the percentage of muscle activation intensity of the two hemi-trunks was calculated.

\subsection{Statistical analysis}

IBM SPSS Statistics ${ }^{\circledR}$ software, version 20.0, (IBM Corporation, Armonk NY, United States of America) was used for the descriptive and inferential data analysis, with a significance level of 0.05 . Shapiro-Wilk test was used to test the normality of the data. The central tendency (mean) and dispersion (standard deviation) measures were used for the descriptive statistics. Repeated Measures Analysis of Variance was used to compare the percentage of muscle activation intensity between the different evaluation tasks (without respiratory load and with inspiratory or expiratory loads), during inspiration and expiration. Bonferroni correction was used for the post-hoc analysis (Marôco, 2014). To quantify the effect size, the partial eta square $\left(\eta_{p}^{2}\right)$ values will be calculated using cut-off provided by Cohen: $\eta_{p}^{2}=0.01$ - small effect, $\eta_{p}^{2}=0.06$ - medium effect, and $\eta_{p}^{2}=0.14$ - large effect (Cohen, 1988).

\section{Results}

\subsection{Inspiration}

During inspiration (Fig. 3), no significant differences were found in the activation intensity of RA an EO muscles between tasks. $\mathrm{TrA} / \mathrm{IO}$ muscle activation intensity was significantly lower with 


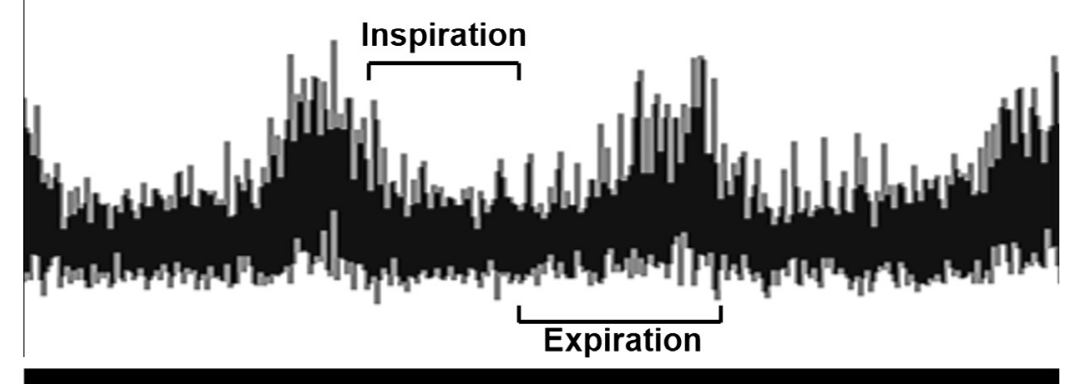

Flow signal

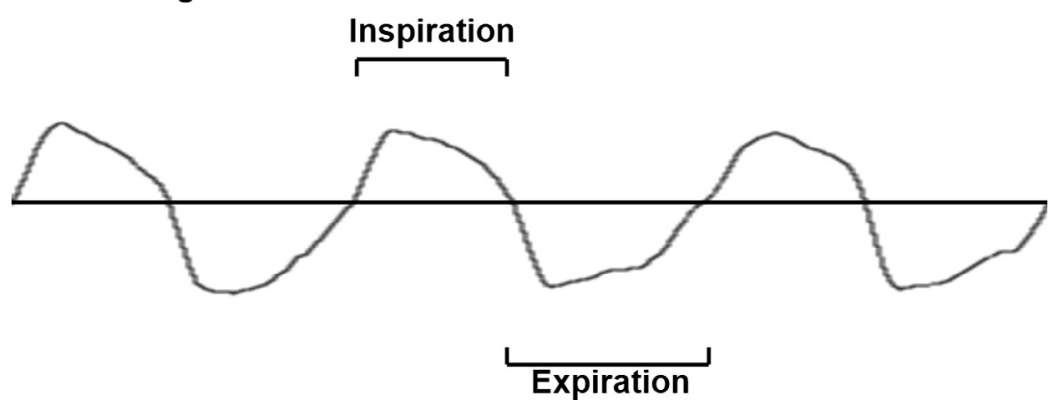

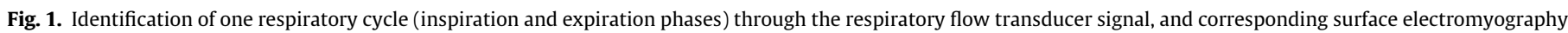
(sEMG) signal analysis.

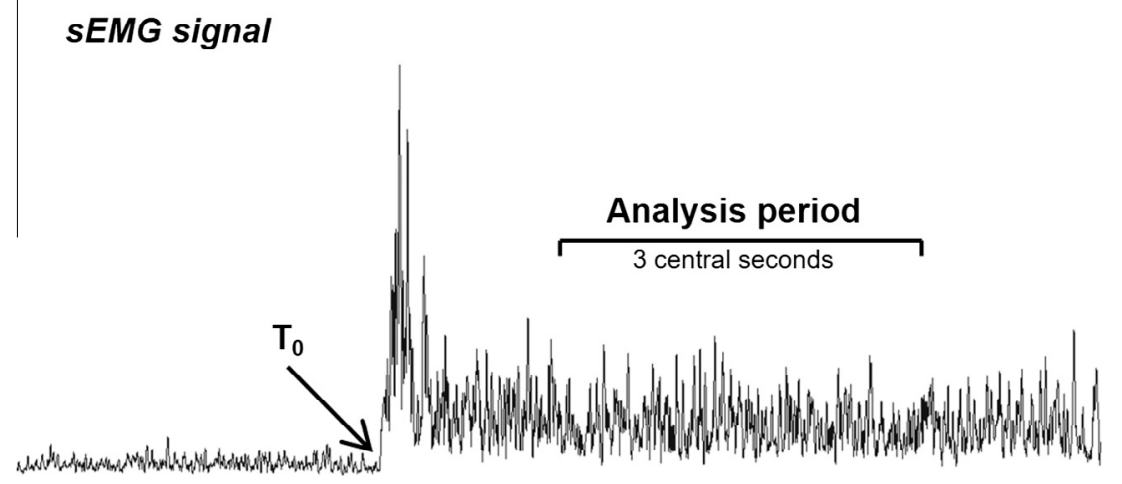

Fig. 2. Surface electromyography (sEMG) signal analysis of one maximal expiratory pressure manoeuvre. $\mathrm{T}_{0}$ is the initial of expiratory phase.

inspiratory load when compared to without respiratory load $(p=0.009)$ and expiratory load $(p=0.002)$.

\subsection{Expiration}

During expiration (Fig. 4), the activation intensity of all abdominal muscles was significantly greater with expiratory load when compared to without respiratory load (RA: $p=0.005$; EO: $p=0.014 ; \operatorname{TrA} / \mathrm{IO}: p<0.001)$. The activation intensity of EO $(p=0.036)$ and $\operatorname{TrA} / \mathrm{IO}(p=0.022)$ was significantly greater with inspiratory load when compared to without respiratory load. TrA/IO muscle activation intensity was significantly greater with expiratory load when compared to inspiratory load $(p<0.001)$.

\subsection{Effect size}

Table 3 show the $\eta_{p}^{2}$, effect size and power test for the comparison of the percentage of activation intensity of RA, EO and TrA/IO muscles between the different tasks, during inspiration and expiration. During inspiration, the comparison between the different tasks showed a large effect in TrA/IO muscle activation intensity $\left(\eta_{p}^{2}=0.248\right)$. Still, during expiration, the comparison between the different tasks showed a large effect in the activation intensity of all abdominal muscles (RA: $\eta_{p}^{2}=0.167 ;$ EO: $\eta_{p}^{2}=0.239 ; \operatorname{Tr} \mathrm{A} / \mathrm{IO}: \eta_{p}^{2}=0.545$ ).

\section{Discussion}

The present study showed that, during inspiration, only $\operatorname{Tr} \mathrm{A} / \mathrm{IO}$ muscle activation intensity was lower with inspiratory load when compared to without respiratory load and expiratory load. During expiration, the activation intensity of abdominal muscles was higher with both inspiratory and expiratory loads. Nevertheless, TrA/IO muscle activation intensity was higher with expiratory load 


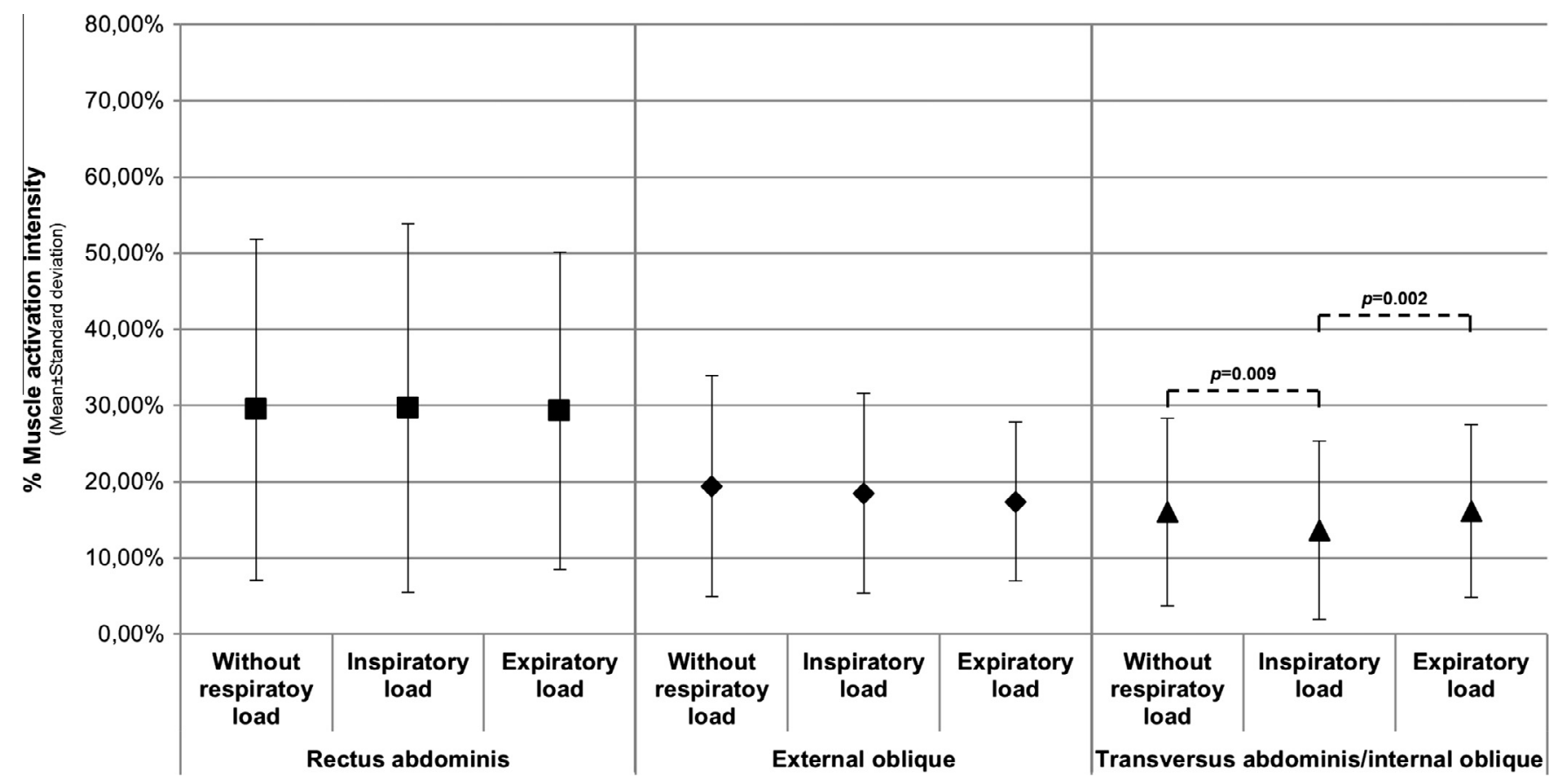

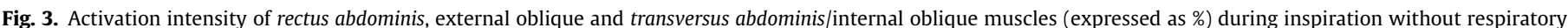
load and with inspiratory or expiratory loads. Data are presented as mean and standard deviation. $p$ values for significant differences between tasks are also presented.

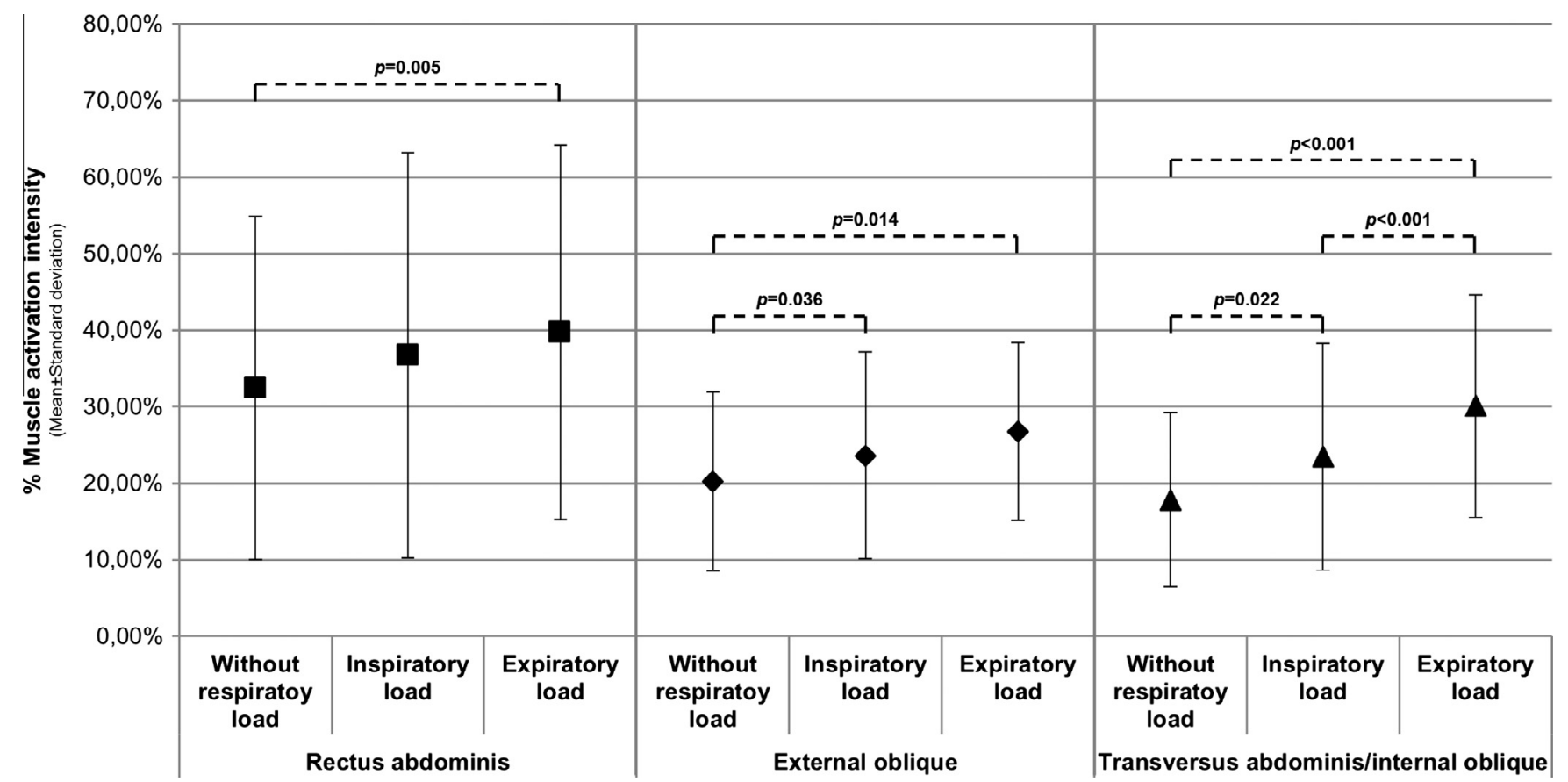

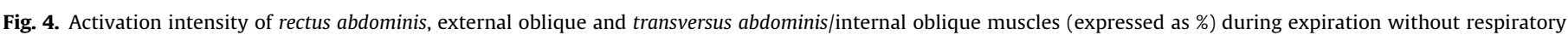
load and with inspiratory or expiratory loads. Data are presented as mean and standard deviation. $p$ values for significant differences between tasks are also presented.

when compared to inspiratory load. These data suggested that the abdominal muscles are important for the breathing mechanics.

All participants breathed at a same rhythm. The quiet expiration is a passive process, involving the use of elastic components recoil of the lungs and chest wall; no phasic activity of abdominal muscles is observed (Kenyon et al., 1997). However, in this study, the breathing with a mandatory rhythm increased the minute ventilation, implying a breathing below functional residual capacity and, consequently, a phasic respiratory activity of abdominal muscles. Moreover, the standardization of respiratory rhythm may have reduced a possible bias related to the respiratory load on minute ventilation.

The outcomes of the present study indicated that the $\operatorname{Tr} \mathrm{A} / \mathrm{IO}$ muscle activation intensity was lower with inspiratory load when compared to without respiratory load and expiratory load, during inspiration, with a large effect. Previous research emphasized that the contribution of abdominal muscles for breathing, apart from their obvious expiratory action, may also be important during 
Table 3

Partial eta squared, effect size and power test for the comparison of the percentage of activation intensity of rectus abdominis (RA), external oblique (EO) and transversus abdominis/internal oblique muscles (TrA/IO) between the different evaluation tasks (without respiratory load and with inspiratory or expiratory loads), during inspiration and expiration.

\begin{tabular}{lllll}
\hline Breathing phase & Muscle & Partial eta squared & Effect size & Power test \\
\hline Inspiration & RA & 0.002 & 0.045 & 0.070 \\
& EO & 0.061 & 0.255 & 0.738 \\
& TrA/IO & 0.248 & 0.574 & 1000 \\
Expiration & RA & 0.167 & 0.448 & 0.997 \\
& EO & 0.239 & 0.560 & 1.000 \\
& TrA/IO & 0.545 & 1.094 & 1.000 \\
\hline
\end{tabular}

inspiration (Macklem, 2014) when the respiratory drive increases, e.g., exercise or mechanical load (Aliverti et al., 1997; De Troyer et al, 1990). For that, breathing is achieved by an alternate modulation of activity of the diaphragm and $\operatorname{TrA}$ muscles, resulting on cyclic changes in the shape of pressurized abdominal cavity. During inspiration, the lower $\operatorname{Tr} \mathrm{A} / \mathrm{IO}$ muscle recruitment seemed to minimize the increased intra-abdominal pressure, as a result of the greater diaphragm muscle recruitment in response to the inspiratory load. Thus, in the present study, the results suggested that although the activity of RA and EO muscles were a non inspiration-related modulation, only the TrA/IO muscle activity was modulated during inspiration to support ribcage and abdominal movements, without losing trunk stability (Hodges and Gandevia, 2000b).

Nevertheless, in this study, the activation intensity of EO and $\mathrm{TrA} / \mathrm{IO}$ muscles with inspiratory load was greater than without respiratory load, during expiration, with a large effect. In fact, the abdominal muscle contraction during expiration contributes to the next inspiration, maintaining the diaphragm muscle closer to its optimal length for tension generation (contractility) (De Troyer and Estenne, 1988). The gradual relaxation of TrA/IO muscle during inspiration observed in the present study minimizes the rib cage distortion and unloads the diaphragm muscle, allowing it to act as a flow generator rather than as a pressure generator (Macklem, 2014). The results of this study were consistent with earlier studies of De Troyer et al. (1990). Aliverti et al. (1997) reported that all abdominal muscles are recruited at the onset of even minimal exercise and their contribution increases progressively as it increases the exercise power. However, Hodges et al. (1997b) reported that only phasic activity of the TrA and IO muscles is observed during expiration with inspiratory load. These authors used a narrow tube to apply the inspiratory load. So, the overload may not be equal between subjects. Still, in the present study, the inspiratory load magnitude was calculated in relation to the MIP value of each participant. Furthermore, during data collection, all participants of this study remained standing, with their knees in loose pack position contrasting to the position used by Hodges et al. (1997b). The increased EO muscle activity due to the greater postural load of non-relaxed position may be a mechanism to compensate the reduced contribution of TrA and diaphragm muscles to the trunk stability. In fact, Hodges et al, (2001) reported that when the respiratory function of diaphragm muscle is challenged, the postural function of this and of TrA muscle may also be challenged, resulting in a negative effect on postural control. Further studies are needed to evaluate the effect of a low inspiratory load on postural control. The specific recruitment of abdominal muscles observed in the present study may provide a mechanism for the CNS to coordinate the respiration and the spinal control during breathing with a low inspiratory load.

All abdominal muscles showed a greater activation intensity with expiratory load when compared to without respiratory load, during expiration, with a large effect. In fact, voluntary efforts, such as empty the lung or raise the intra-abdominal pressure, are achieved by the contraction in concert of the superficial (RA and EO muscles) and deep (TrA/IO muscle) muscle layers of ventrolateral abdominal wall (De Troyer et al., 1990). These findings are corroborated by Barrett et al. (1994), who found that the abdominal muscle recruitment increases in proportion to the expiratory threshold load. Kaneko et al. (2006) also reported an increased abdominal muscle recruitment (thickness), measured by ultrasonography. Nevertheless, De Troyer et al. (1990) and Goldman et al. (1987) found that the $\operatorname{TrA}$ muscle frequently contracts together with EO and RA muscles during expiration below functional residual capacity, voluntary cough, expulsive manoeuvres and speech. Furthermore, in the present study, the activation intensity of $\operatorname{Tr} \mathrm{A} / \mathrm{IO}$ muscle was greater with expiratory load when compared with inspiratory load, during expiration. Despite the fact that all abdominal muscles tend to contract together during expiratory voluntary efforts, there seems to be a specific recruitment of TrA/IO muscle, regarding RA and the EO muscles, during breathing with expiratory load. These findings are consistent with earlier studies of Kaneko et al. (2006), who reported that the expiratory threshold loading increases mainly the TrA muscle thickness. This specific recruitment of the $\mathrm{Tr} A / \mathrm{IO}$ muscle strengthens the knowledge that the effect of this muscle on abdominal pressure is important to the act of breathing.

All participants in this study did not suffer from chronic respiratory pathologies. Chronic obstructive pulmonary disease may alter the patient's ability to modulate their abdominal muscles recruitment to maintaining the dual task. Therefore, it is important to understand if the abdominal muscle synergy observed with a low inspiratory load, it would be present in this population, for allow breathing without loosing the trunk stability. Otherwise, the results of the present study suggested that only $10 \%$ of MEP can have a potential impact on the facilitation of abdominal muscle activation, namely $\mathrm{Tr} \mathrm{A} / \mathrm{IO}$ muscle, and, consequently, improves the breathing mechanics, minimizing the rib cage distortion and unloading the diaphragm muscle. Furthermore, it is difficult to evaluate the energetic cost of the benefit of sharing the work of abdominal muscles for the breathing. However, the TrA/IO muscle expiratory contraction, due to its circumferential arrangement, should be more effective in increasing abdominal pressure and deflating the lung so as to take on portion the work of the inspiratory muscles (De Troyer et al., 1990). Further studies conducted among chronic obstructive pulmonary disease patients are needed to select the appropriate intervention strategies to optimize the abdominal muscle synergy for the postural and respiratory functions.

\section{Conclusion}

In healthy subjects, the inspiratory and expiratory loads promoted a different impact on abdominal muscle activity during both phases of breathing. Despite all abdominal muscles are important for breathing mechanics, $\mathrm{TrA} / \mathrm{IO}$ muscle recruitment seems to be the most relevant to modulate the intra-abdominal pressure in situations wherein the respiratory demand increases. 


\section{References}

Aliverti, A., Cala, S.J., Duranti, R., Ferrigno, G., Kenyon, C.M., Pedotti, A., et al., 1997. Human respiratory muscle actions and control during exercise. J. Appl. Physiol. $83,1256-1269$.

American Thoracic Society/European Respiratory S, 2002. ATS/ERS statement on respiratory muscle testing. Am. J. Respir. Crit. Care Med. 166, 518-624.

Balasubramaniam, R., Wing, A.M., 2002. The dynamics of standing balance. Trends Cogn. Sci. 6, 531-536.

Barrett, J., Cerny, F., Hirsch, J.A., Bishop, B., 1994. Control of breathing patterns and abdominal muscles during graded loads and tilt. J. Appl. Physiol. 76, 2473-2480.

Beith, I.D., Synnott, R.E., Newman, S.A., 2001. Abdominal muscle activity during the abdominal hollowing manoeuvre in the four point kneeling and prone positions. Man Ther. 6, 82-87.

Chanthapetch, P., Kanlayanaphotporn, R., Gaogasigam, C., Chiradejnant, A., 2009. Abdominal muscle activity during abdominal hollowing in four starting positions. Man Ther. 14, 642-646.

Cholewicki, J., Juluru, K., McGill, S.M., 1999. Intra-abdominal pressure mechanism for stabilizing the lumbar spine. J. Biomech. 32, 13-17.

Cohen, J., 1988. Statistical Power Analysis for the Behavioral Sciences, second ed. L. Erlbaum Associates, Hillsdale, NJ.

Criswell, E., 2011. Cram's introduction to surface electromyography, second ed. Jones \& Bartlett Publishers, Sudbury, MA.

David, P., Laval, D., Terrien, J., Petitjean, M., 2012. Postural control and ventilatory drive during voluntary hyperventilation and carbon dioxide rebreathing. Eur. J. Appl. Physiol. 112, 145-154.

De Troyer, A., Estenne, M., 1988. Functional anatomy of the respiratory muscles. Clin. Chest Med. 9, 175-193.

De Troyer, A., Estenne, M., Ninane, V., Van Gansbeke, D., Gorini, M., 1990 Transversus abdominis muscle function in humans. J. Appl. Physiol. 68, 10101016.

Goldman, J.M., Lehr, R.P., Millar, A.B., Silver, J.R., 1987. An electromyographic study of the abdominal muscles during postural and respiratory manoeuvres. J. Neurol. Neurosurg. Psychiatry 50, 866-869.

Hermens, H.J., Freriks, B., Disselhorst-Klug, C., Rau, G., 2000. Development of recommendations for SEMG sensors and sensor placement procedures. J. Electromyogr. Kinesiol. 10, 361-374.

Hodges, P.W., 1999. Is there a role for transversus abdominis in lumbo-pelvic stability? Man Ther. 4, 74-86.

Hodges, P.W., Butler, J.E., McKenzie, D.K., Gandevia, S.C., 1997a. Contraction of the human diaphragm during rapid postural adjustments. J. Physiol. 505 (Pt 2), 539-548.

Hodges, P.W., Gandevia, S.C., 2000a. Activation of the human diaphragm during a repetitive postural task. J. Physiol. 522 (Pt 1), 165-175.

Hodges, P.W., Gandevia, S.C., 2000b. Changes in intra-abdominal pressure during postural and respiratory activation of the human diaphragm. J. Appl. Physiol. 89, 967-976.

Hodges, P.W. Gandevia, S.C., Richardson, C.A., 1997b. Contractions of specific abdominal muscles in postural tasks are affected by respiratory maneuvers. J. Appl. Physiol. 83, 753-760.

Hodges, P.W., Gurfinkel, V.S., Brumagne, S., Smith, T.C., Cordo, P.C., 2002 Coexistence of stability and mobility in postural control: evidence from postural compensation for respiration. Exp. Brain Res. 144, 293-302.

Hodges, P.W., Heijnen, I., Gandevia, S.C., 2001. Postural activity of the diaphragm is reduced in humans when respiratory demand increases. J. Physiol. 537, 9991008.

Horak, F.B., Henry, S.M., Shumway-Cook, A., 1997. Postural perturbations: new insights for treatment of balance disorders. Phys. Ther. 77, 517-533.

Kaneko, H., Sato, H., Maruyama, H., 2006. Evaluation of lateral abdominal muscle activity during expiratory threshold loading by ultrasonography. J. Phys. Ther. Sci. 18, 187-191.

Kenyon, C.M., Cala, S.J., Yan, S., Aliverti, A., Scano, G., Duranti, R., et al., 1997. Rib cage mechanics during quiet breathing and exercise in humans. J. Appl. Physiol. 83, $1242-1255$.

Kuznetsov, N.A., Riley, M.A., 2012. Effects of breathing on multijoint control of center of mass position during upright stance. J. Mot. Behav, 44, 241-253.

Lackner, J.R., DiZio, P., 2005. Vestibular, proprioceptive, and haptic contributions to spatial orientation. Annu. Rev. Psychol. 56, 115-147.

Macklem, P.T. 2014. The act of breathing. In: Aliverti, A., Pedotti, A. (Eds.) Mechanics of Breathing: New Insights From New Technologies. Springer Milan, Milano, pp. 3-10.

Marôco, J., 2014. Análise Estatística Com o SPSS Statistics, sixth ed. ReportNumber, Lda, Pero Pinheiro.

Marshall, P., Murphy, B., 2003. The validity and reliability of surface EMG to assess the neuromuscular response of the abdominal muscles to rapid limb movement. J. Electromyogr. Kinesiol. 13, 477-489.

Mew, R., 2009. Comparison of changes in abdominal muscle thickness between standing and crook lying during active abdominal hollowing using ultrasound imaging. Man Ther. 14, 690-695.

Reeve, A., Dilley, A., 2009. Effects of posture on the thickness of transversus abdominis in pain-free subjects. Man Ther. 14, 679-684.

Thompson, P.D., 2014. Health appraisal and risk assessment. In: Pescatello, L.S., Arena, R., Riebe, D., Thompson, P.D. (Eds.), ACSM's Guidelines for Exercise Testing and Prescription, ninth ed. Wolters Kluwer/Lippincott Williams \& Wilkins Health, Philadelphia, pp. 1-18. 